\title{
Endoscopic Ultrasound Elastography as a Novel Diagnostic Method for the Assessment of Hardness and Depth of Invasion in Colorectal Neoplasms
}

\author{
Masaya Esaki ${ }^{a}$ Takeshi Yamamurab $^{b}$ Masanao Nakamurab Keiko Maeda ${ }^{a}$ \\ Tsunaki Sawada $^{a}$ Yasuyuki Mizutani $^{\mathrm{b}}$ Eri Ishikawa $^{\mathrm{b}}$ Hiroto Suzuki $^{\mathrm{b}}$ \\ Takeshi Kuno $^{b}$ Kentaro Yamada ${ }^{b}$ Issei Hasegawab Takuya Ishikawab \\ Naomi Kakushima ${ }^{b}$ Kazuhiro Furukawa ${ }^{b}$ Eizaburo Ohno $^{\text {b }}$ Hiroki Kawashima $^{a}$ \\ Yoshiki Hirookac Mitsuhiro Fujishiro ${ }^{\mathrm{b}}$ \\ aDepartment of Endoscopy, Nagoya University Hospital, Nagoya, Japan; ${ }^{\text {b}}$ Department of Gastroenterology and \\ Hepatology, Nagoya University Graduate School of Medicine, Nagoya, Japan; 'Department of Gastroenterology and \\ Gastroenterological Oncology, Fujita Health University School of Medicine, Toyoake, Japan
}

\begin{abstract}
Keywords
Colorectal neoplasm - Endoscopic ultrasound - Endoscopic ultrasound elastography · Magnifying chromoendoscopy
\end{abstract}

\begin{abstract}
Introduction: We aimed to compare the efficacy of endoscopic ultrasound elastography (EUS-EG) with that of magnifying chromoendoscopy (MCE) and endoscopic ultrasonography (EUS) for the diagnosis of the depth of invasion in colorectal neoplasms. This is an important clinical issue as the depth of invasion is associated with the risk of metastasis. Methods: Consecutive patients with suspected superficial colorectal neoplasms, evaluated by MCE, EUS, and EUSEG, for whom endoscopic submucosal dissection was considered, were enrolled in 2018 (derivation study) and in 2019-2020 (validation study). The primary clinical endpoint was the diagnostic yield differentiating intramucosal and shallow submucosal neoplasms from deep submucosal (dSM) and advanced colorectal cancers. In addition, interand intra-observer agreements of the elastic score of colorec-
\end{abstract}

tal neoplasm (ES-CRN) were evaluated by 2 expert and 2 non-expert endoscopists. Results: Thirty-one (33 lesions) and 50 (55 lesions) patients were enrolled in the derivation and validation studies, respectively. Sensitivity, specificity, positive, and negative predictive values, and accuracy of assessment of the depth of submucosal or deeper invasion in the derivation and validation groups were as follows: EUSEG, 100/88.2/86.7/100/93.3\%and $77.8 / 86.1 / 73.7 / 88.6 / 83.3 \%$; MCE, $66.7 / 94.4 / 90.9 / 77.3 / 81.8 \%$ and $84.2 / 91.4 / 84.2 / 91.4 /$ 88.9\%; and EUS, 93.3/77.8/77.8/93.3/84.8\% and 89.5/65.7/ $58.6 / 92.0 / 74.1 \%$, respectively. For the 2 expert endoscopists, interobserver agreement for the ES-CRN (first and second assessments) in the derivation group was 0.84 and 0.78 , respectively; these values were 0.73 and 0.49 , respectively, for the 2 non-expert endoscopists. Discussion/Conclusion: All 3 modalities presented similar diagnostic yield. Inter- and intra-observer agreements of the ES-CRN were substantial, even for non-expert endoscopists. Therefore, EUS-EG may be a useful modality in determining the depth of invasion in colorectal neoplasms.

(c) 2020 The Author(s)

Published by S. Karger AG, Basel karger@karger.com www.karger.com/dig

Karger $\stackrel{\text { ' }}{5}$

BOPEN ACCESS
(C) 2020 The Author(s)

Published by S. Karger AG, Basel

This is an Open Access article licensed under the Creative Commons Attribution-NonCommercial-4.0 International License (CC BY-NC) (http://www.karger.com/Services/OpenAccessLicense), applicable to the online version of the article only. Usage and distribution for commercial purposes requires written permission.
Takeshi Yamamura

Department of Gastroenterology and Hepatology Nagoya University Graduate School of Medicine 65 Tsuruma-cho, Showa-ku, Nagoya 466-8550 (Japan) tyamamu@med.nagoya-u.ac.jp 


\section{Introduction}

Endoscopic resection, including endoscopic mucosal resection and endoscopic submucosal dissection (ESD), is widely used to treat colorectal neoplasms with invasion limited to the mucosa or shallow submucosa [1-3]. Appropriate diagnosis of intramucosal colorectal neoplasms is important, as these lesions have negligible risk for lymph node metastasis $[4,5]$. However, submucosal invasive colorectal carcinoma has a risk for lymph node metastasis of $10-15 \%$ [6-8]. The rate of lymph node metastasis for submucosal invasive colorectal carcinomas is non-uniform and being dependent on the depth of submucosal invasion and other factors. When the submucosal invasion depth is limited to $<1,000 \mu \mathrm{m}$, without lymphatic or venous invasion, sprouting, or poorly differentiated components, the rate of lymph node metastasis is approximately $0 \%$ [5]; thus, endoscopic resection would be indicated.

Currently, the depth of invasion of colorectal neoplasms is estimated using magnifying endoscopy (ME) with image enhancement, including the optical digital method [9-11], dye-spraying method [12-14], and endoscopic ultrasonography (EUS) [15-17]. However, predicting the depth of invasion remains difficult in some cases, particularly for protruded-type lesions $[18,19]$. In these cases, another imaging modality might be necessary, such as EUS elastography (EUS-EG) which quantifies the relative hardness (or elasticity) of different tissues [20]. EG has been effectively used to identify neoplastic lesions in the breast, pancreas, prostate, lymph nodes, and rectum [20-27]. To date, a few studies have reported on the use of elastography to evaluated rectal neoplasms $[27,28]$. However, the use of elastography with echoendoscopic assessment of whole colorectal neoplasms and, in particular, for early colorectal cancer, has not been previously clarified. Moreover, elastic scores for the colorectum have not yet been determined. As such, the applicability of elastography to diagnose the depth of invasion colorectal neoplasms has not been evaluated. Accordingly, our aim in this study was to compare the efficacy of EUS-EG with that of EUS and magnifying chromoendoscopy (MCE) for the diagnosis of the depth of invasion of colorectal neoplasms.

\section{Materials and Methods}

\section{Selection of Patients}

Consecutive patients who underwent colonoscopy and echoendoscopy for suspected superficial colorectal neoplasms for whom ESD was considered, at Nagoya University Hospital, Japan, between March and December 2018, were eligible for the derivation study. Consecutive patients meeting these criteria, between January 2019 and January 2020, were eligible for the validation study. Exclusion criteria were as follows: age $>90$ years (as superold patients are more likely to have unexpected events which would increase the procedure time); a lesion size for the target lesions for endoscopic assessment of $<20 \mathrm{~mm}$, scarring of lesions (subjective assessment at the time of the examination); inflammatory bowel diseases; and pregnancy. All patients had undergone examination using conventional white light imaging (WLI) endoscopy and MCE (MCE; CF-HQ290ZI; Olympus Medical Systems Corp., Tokyo, Japan or EC-600ZP7; Fujifilm Co., Tokyo, Japan) before EUS-EG. EUS-EG was performed using a forward-viewing radial-array echoendoscope (EG-580UR; Fujifilm Co., Ltd; see online suppl. Table; for all online suppl. material, see www.karger. com/doi/10.1159/000511589). Endoluminal carbon dioxide $\left(\mathrm{CO}_{2}\right)$ insufflation was used for all patients during the endoscopic procedure $\left(\mathrm{CO}_{2}\right.$ gas supply system $\mathrm{GW}-100$; Fujifilm Co., Tokyo, Japan). All lesions were treated using endoscopic or surgical resection within 1 month of imaging, with the final diagnosis confirmed histopathologically.

\section{Principles of EUS-EG}

In elastography, tissue strain, which is negatively correlated with tissue elasticity, is generated by applying slight manual compression and comparing the data obtained before and after compression. The data are compared using a cross-correlation technique to determine the amount of displacement each small portion of tissue undergoes in response to the compression applied by the ultrasound transducer $[29,30]$. The elasticity modulus can be calculated from the strain and stress on the structures examined. EUS-EG provides a relative value of strain in a specific region of interest (ROI). Tissue strain is expressed with color maps on a Bmode image, with soft tissue expressed in red and hard tissue in blue. Based on the fact that a tumor becomes fibrotic and harder as it invades deeper tissues, in this study, we examined whether the invasion depth could be diagnosed by comparing the hardness of the tumor relative to the surrounding tissue.

\section{EUS and EUS-EG Settings}

All EUS procedures were performed by experienced echoendoscopists (M.E. and T.K.), and each having conducted $>100$ EUS and $>3,000 \mathrm{ME}$ procedures of the colon. The echoendoscope was inserted through the anus, following standard colonoscopy, with patient positioning adjusted to place the lesion under gravity. EUS was performed with the patient under sedation (intravenous administration of $0.05 \mathrm{mg} / \mathrm{kg}$ midazolam for induction and additional $1 \mathrm{mg}$ doses, as needed, for restlessness and body movement during the procedure), with heart rate, blood pressure, and hemoglobin oxygen saturation $\left(\mathrm{SpO}_{2}\right)$ monitored. An antispasmodic agent (butylscopolamine, $20 \mathrm{mg}$ or glucagon, and $0.5 \mathrm{or} 1 \mathrm{mg}$ ) was administered immediately before inserting the endoscope, unless contraindicated based on the patient's medical history. An additional dose of $10 \mathrm{mg}$ of butylscopolamine or $0.5 \mathrm{mg}$ of glucagon was administered in the presence of strong bowel peristalsis. Overall, antispasmodic agents were not used in 10 patients; glucagon was used in 8 patients; and butylscopolamine in the remaining 63.

Insufflated $\mathrm{CO}_{2}$ was removed to decompress the bowel lumen, and deaerated water was infused using an irrigation pump (UWS1; Olympus Optical Co., Ltd., Tokyo, Japan). Observation was per- 


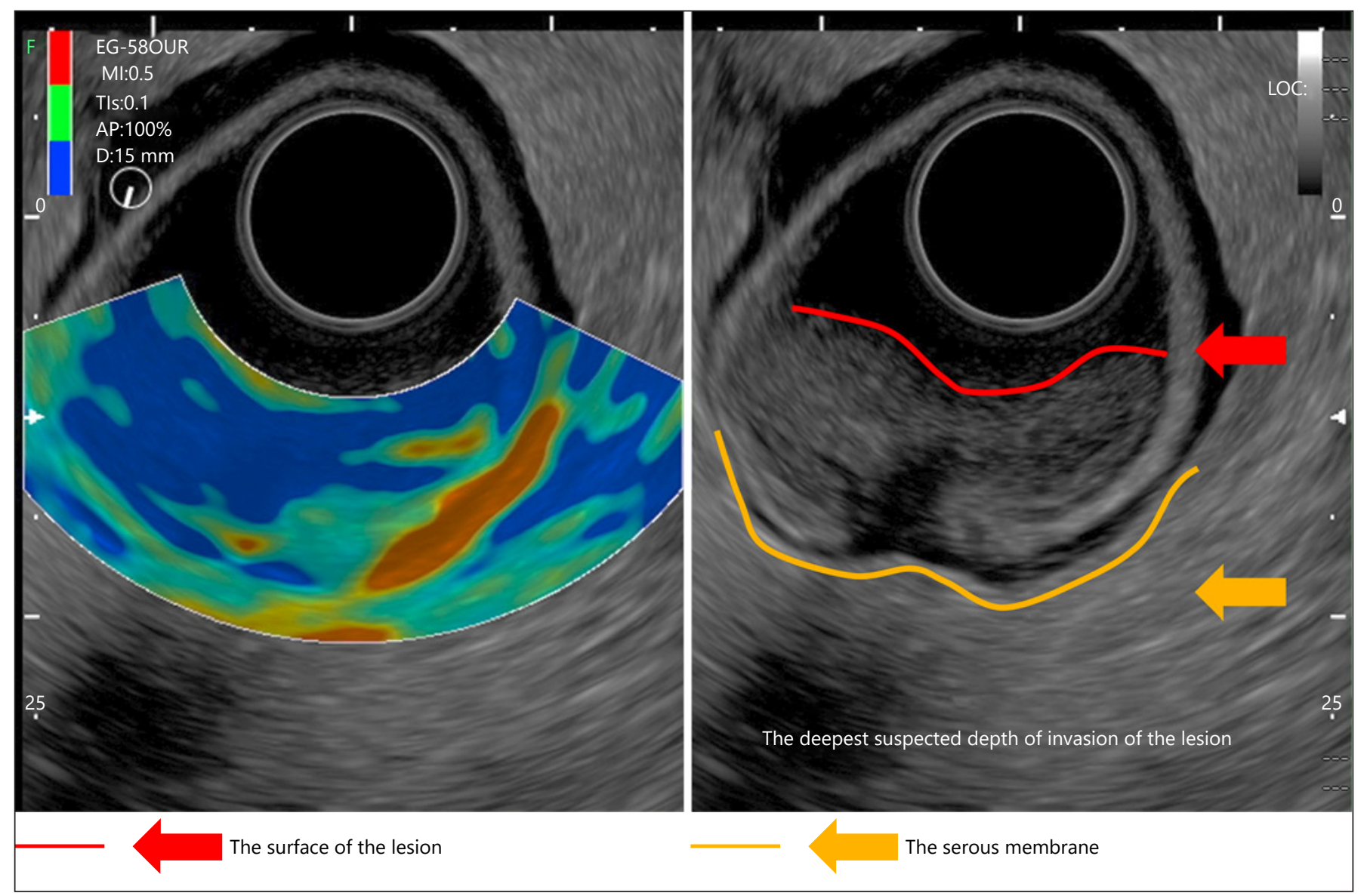

Fig. 1. Setting of the ROI for EUS-EG of colorectal neoplasms. ROI, region of interest; EUS-EG, endoscopic ultrasound elastography.

formed while replenishing water, as appropriate. The EG-580UR echoendoscope (Fujifilm Co., Ltd.), equipped with a Sonart SU-1 ultrasound processor (Fujifilm Co., Ltd.), was used in all cases. EUS-EG was performed in the radial scanning mode, with lesions identified under B-mode observation, at $12-\mathrm{MHz}$ for both signal transmission and reception.

The ROI was selected to be as close as possible to the target lesion size, with the area from the surface of the lesion to the serous membrane included for evaluating tissue elasticity. The location was set to the deepest suspected depth of invasion of the lesion (Fig. 1). The echoendoscope was fixed on the lesion for a few seconds to obtain a stable image which is required for elastic pattern definition. From a cine-loop of the EUS-EG imaging obtained, all lesion images were stored as still images (bitmap format) for offline analysis. The elastic score of the pancreas was used as the reference tissue hardness for calibration.

\section{Analysis of EUS-EG Images}

EUS-EG images were classified into 3 patterns using the elastic score of colorectal neoplasm (ES-CRN; Fig. 2) as follows: ES-CRN 1 , the tumor region is softer (green, yellow, and red coloring) than the surrounding area (blue); ES-CRN 2, the tumor region is hard- er (blue) or as hard (blue) as the surrounding area; and ES-CRN 3, a honeycomb-like pattern is observed within the lesion. ES-CRN 1 lesions were defined as having less than slight submucosal (sSM) invasion and ES-CRN 2 and 3 lesions as having deeper submucosal invasion. The EUS-EG-derived measure of the hardness of the tumor was compared to that of the muscle layer within the ROI; if the tumor had a hardness equal to or higher than that of the muscle layer, the tumor mainly turns blue. The tumors were classified based on a review of the color-coded images obtained by experienced echoendoscopists in the derivation study.

\section{Reliability of the ES-CRN Classification}

Reliability of the ES-CRN classification was evaluated in addition to the derivation study between 2 expert (T.Y. and H.S.) and 2 non-expert (I.H. and K.Y.) endoscopists who were blinded to information regarding WLI, MCE, EUS, and the final histological diagnosis. Expert endoscopists had each performed $>3,000 \mathrm{ME}$ and $>100$ EUS procedures of the colon and had observed EUS-EG procedures. Non-experts had each performed $>3,000 \mathrm{ME}$ procedures but had neither observed nor performed EUS-EG procedures and had limited experience performing EUS of the colon. EUS-EG images were reviewed after a short lecture on the ES-CRN 

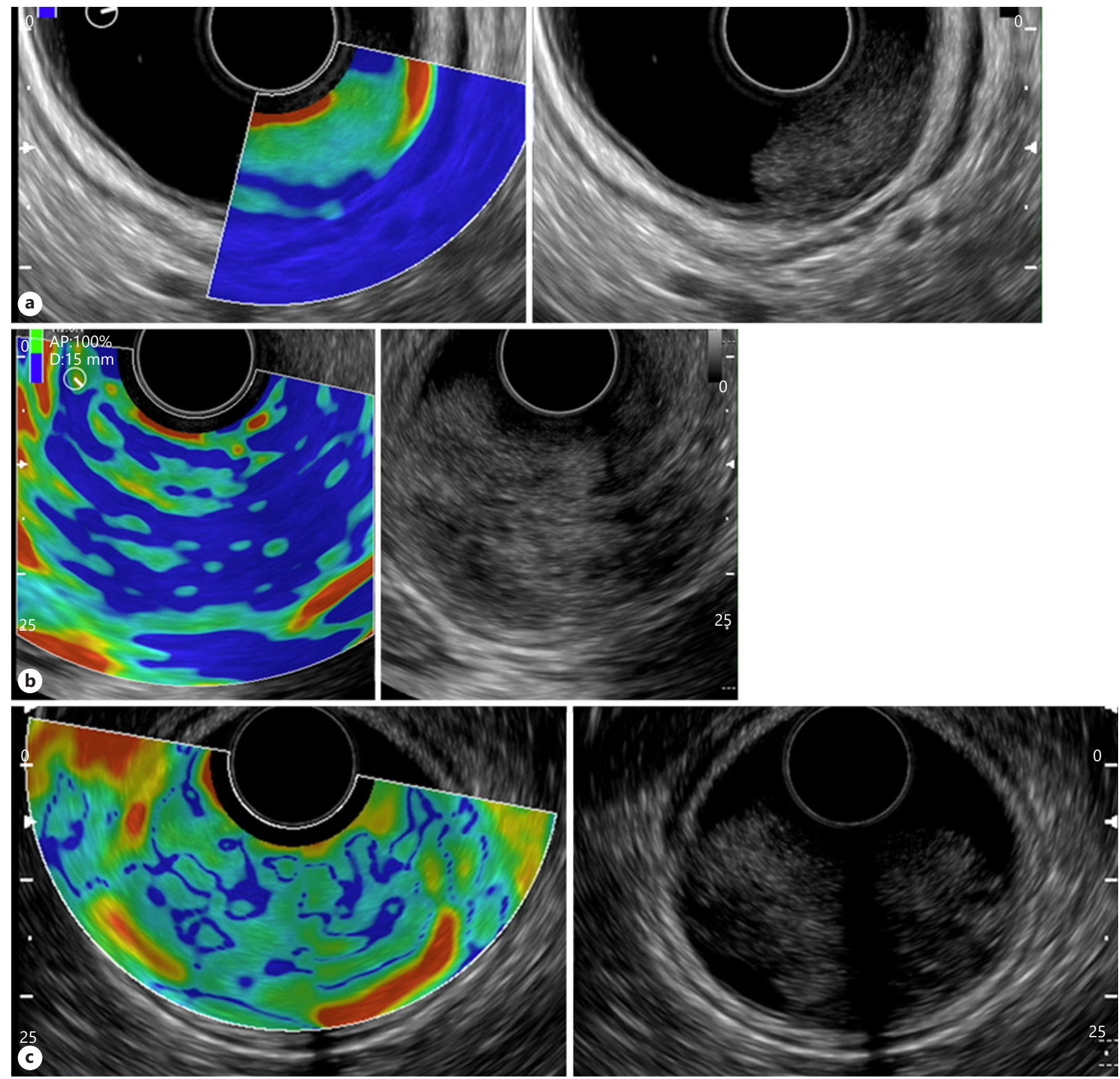

Fig. 2. Elastic score of colorectal neoplasm (ES-CRN). a ES-CRN 1: the lesion is mostly in the soft tissue range (green, yellow, and red), with the hard tissue range (blue) being almost exclusive to the muscularis propria (MP; fourth layer of B mode). $\mathbf{b}$ ES-CRN 2: the lesion is mostly in the hard tissue range (blue). c ES-CRN 3: honeycomblike pattern within the lesion. ES-CRN, elastic score of colorectal neoplasm; MP, muscularis propria.

classification, with reassessment 2 weeks after, using a random order of the same images.

Inter- and intra-observer agreements were calculated using the Kappa coefficient, interpreted as follows: poor, 0-0.20; fair, 0.210.40; moderate, 0.41-0.60; substantial, 0.61-0.80; and excellent, 0.81-1.00 [31]. Notably, experienced echoendoscopists who per- formed EUS-EG were excluded from the process of reliability examination.

\section{Analysis of MCE and EUS Images}

MCE images were diagnosed using the pit pattern [12]. The index for dSM or deeper invasion on MCE was defined by VI high- 


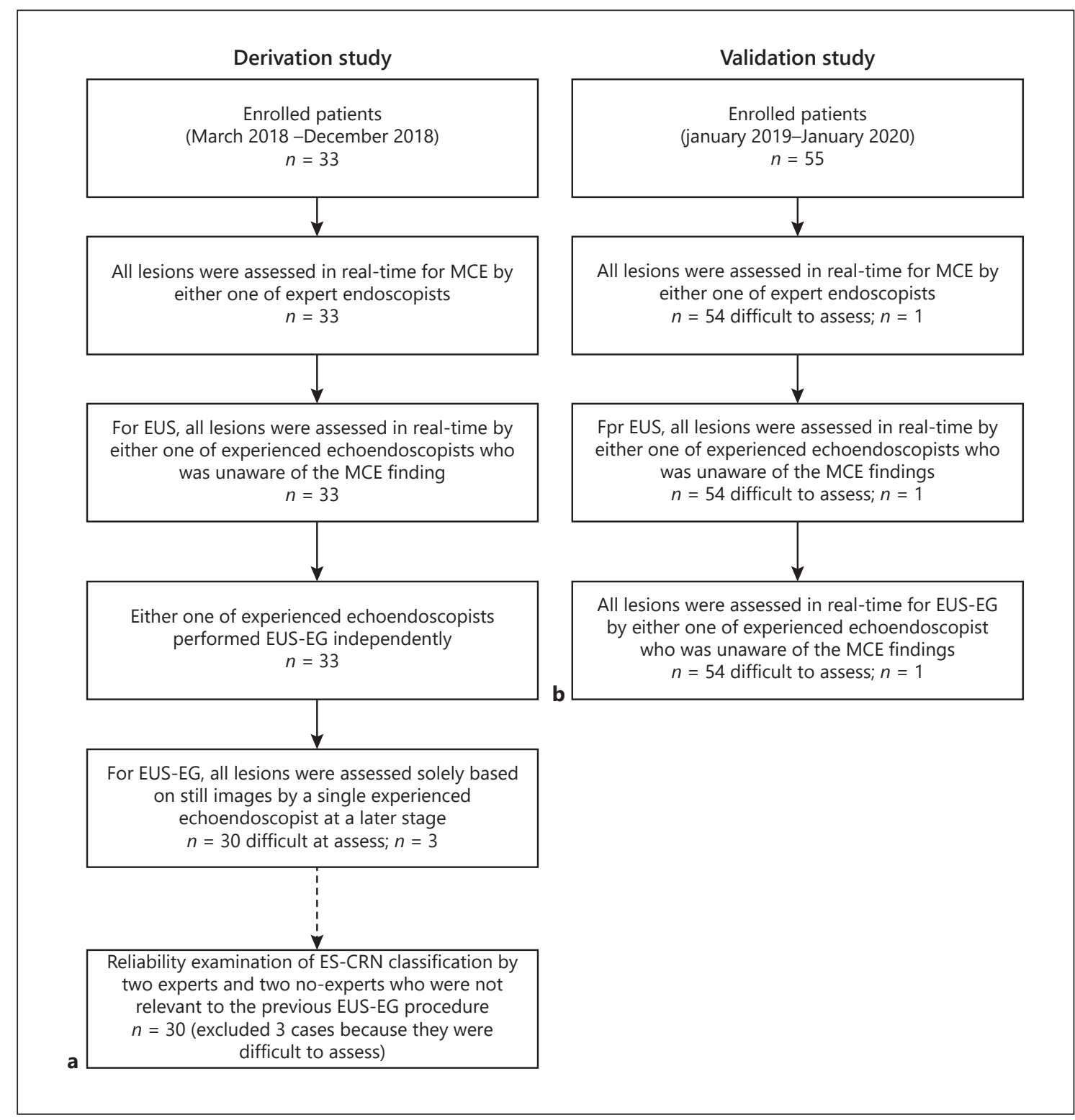

Fig. 3. Study flow chart: a derivation study, b validation study. EUS-EG, endoscopic ultrasound elastography, ES-CRN, elastic score of colorectal neoplasm.

grade or VN pit pattern. In comparison with the ES-CRN classification, the depth of invasion recorded during MCE and EUS examination was used, with no further verification of images.

\section{Assessment of Diagnosis Using Each Modality}

In both of the derivation and the validation studies, lesions difficult to evaluate by each modality were excluded. The lesions difficult to evaluate were judged at the time of diagnosis for each modality. All remaining lesions were assessed in real time by either 1 of the 2 expert endoscopists (T.Y., H.S.) for MCE and by either 1 of the experienced echoendoscopists (M.E., T.K.) for EUS. All analyses were performed without knowledge of the MCE findings.
In the derivation study, for EUS-EG, all lesions were diagnosed solely from still images by either one of the experienced echoendoscopists. These lesions were then assessed by 4 doctors ( 2 experts and 2 non-experts) to determine the reliability of assessment. In the validation study, all lesions were assessed in real time for EUSEG by either one of the experienced echoendoscopists, without knowledge of the MCE findings (Fig. 3). The diagnostic ability was calculated with exclusion of cases difficult to assess for all modalities. The diagnosis of EUS-EG was assigned a level of confidence (high or low) based on predictive accuracy, with a $>90 \%$ accuracy classified as a diagnosis with "high confidence" [32]. 
Table 1. Patients' characteristics and indications for EUS-EG

\begin{tabular}{|c|c|c|}
\hline Patients' characteristics & $\begin{array}{l}\text { Derivation study, } \\
n=33\end{array}$ & $\begin{array}{l}\text { Validation study, } \\
n=55\end{array}$ \\
\hline$N($ male/female $)$ & $33(20 / 13)$ & $55(29 / 26)$ \\
\hline Age, median (range), years & $70(50-87)$ & $70(34-85)$ \\
\hline \multicolumn{3}{|l|}{ Tumor location } \\
\hline $\mathrm{R} / \mathrm{S} / \mathrm{D} / \mathrm{T} / \mathrm{A} / \mathrm{C}, n$ & $12 / 10 / 0 / 5 / 5 / 1$ & $22 / 10 / 5 / 4 / 9 / 5$ \\
\hline Tumor size, median (range), mm & $36(15-100)$ & $25(10-82)$ \\
\hline \multicolumn{3}{|l|}{ Morphology type, $n$} \\
\hline Is/Ip/IIa/Is + IIa/Is + IIc/IIa + Is/IIa + IIc & $9 / 5 / 3 / 4 / 1 / 7 / 4$ & $16 / 1 / 17 / 7 / 0 / 6 / 8$ \\
\hline Endoscopic/surgical resection, $n$ & $20 / 13$ & $35 / 20$ \\
\hline \multicolumn{3}{|l|}{$\begin{array}{l}\text { Depth of final diagnosis of histopathological assessment, } \\
n \mathrm{~m} / \mathrm{sSM} / \mathrm{dSM} / \mathrm{MP} / \mathrm{SS}\end{array}$} \\
\hline \multicolumn{3}{|l|}{ Insertion time, median (range), $s$} \\
\hline Total & $60(5-900)$ & $60(1-1,800)$ \\
\hline \multicolumn{3}{|l|}{ Lesion site } \\
\hline Rectum & $5(5-30)$ & $5(1-60)$ \\
\hline Sigmoid colon & $75(10-300)$ & $42(15-120)$ \\
\hline Descending colon & na & $180(62-780)$ \\
\hline Transverse colon & $180(120-600)$ & $390(120-570)$ \\
\hline Ascending colon & $420(180-900)$ & $270(120-380)$ \\
\hline Cecum & 600 & $420(178-1,800)$ \\
\hline
\end{tabular}

$\mathrm{R}$, rectum; S, sigmoid colon; $\mathrm{D}$, descending colon; $\mathrm{T}$, transverse colon; $\mathrm{A}$, ascending colon; $\mathrm{C}$, cecum; $\mathrm{m}$, intramucosal cancer; sSM, slight submucosal; dSM, deep submucosal; EUS-EG, endoscopic ultrasound elastography; MP, muscularis propria; SS, subserosa; na, not applicable; $N$, number.

\section{Histopathological Assessment}

Resected specimens were immediately placed in formalin and embedded in paraffin wax for histological examination. Pathological diagnoses were established by experienced pathologists, on the basis of hematoxylin-eosin and immunopathological staining. The vertical depth of submucosal invasion was defined as $<1,000$ and $\geq 1,000 \mu \mathrm{m}$ as sSM and dSM invasion, respectively [5].

\section{Outcome Measures}

Primary Endpoint: Diagnostic Ability

The primary endpoint of this study was the diagnostic ability. Sensitivity, specificity, positive predictive value (PPV), negative predictive value (NPV), and accuracy of the ES-CRN classification and MCE/EUS diagnosis yield for a dSM or deeper lesion were compared.

\section{Secondary Outcomes: Clinical Efficacy}

Diagnostic performance for each ES-CRN classification was evaluated. Inter- and intra-observer agreements between the 2 expert and 2 non-expert endoscopists were evaluated. Technical feasibility was defined as the time from insertion of the echoendoscope into the anus to arrival at the target lesion. Adverse events were defined as any deviation from the clinical course after EUSEG, as observed by the 2 expert endoscopists. All patients were contacted within 1 month of the procedure to determine the presence of any late-onset adverse events.

\section{Sample Size Calculation}

For dSM or deeper lesions, pooled estimates from a meta-analysis of MCE and reported a sensitivity and specificity of 81 and 95\%, respectively [33]. Assuming that EUS-EG has the equivalent diagnostic ability as MCE, we set an accuracy $>80 \%$ as necessary criterion. A power analysis indicated that 23 and 51 lesions were needed to achieve this criterion in the derivation and the validation studies, based on a 20 and 5\% significance level for the derivation and validation study, respectively, a statistical power of $80 \%$, and an effect size of 0.5 , using a 1 -sided equivalence. Therefore, 33 and 55 lesions were estimated to be needed, accounting for a reasonable anticipated dropout rate, in the derivation and the validation study, respectively.

\section{Statistical Analysis}

Continuous variables, including patients' age and lesion size, were presented as median and range. Sensitivity, specificity, PPV, and NPV were calculated for the EUS-EG-, MCE-, and EUS-based classification of lesions, using the histopathological diagnosis as the reference. Inter- and intra-observer agreements for the EUSEG classification were calculated using the Kappa coefficient. Insertion time was reported as median and interquartile range. All analyses were performed using SPSS software (version 24; SPSS Japan, Tokyo). 

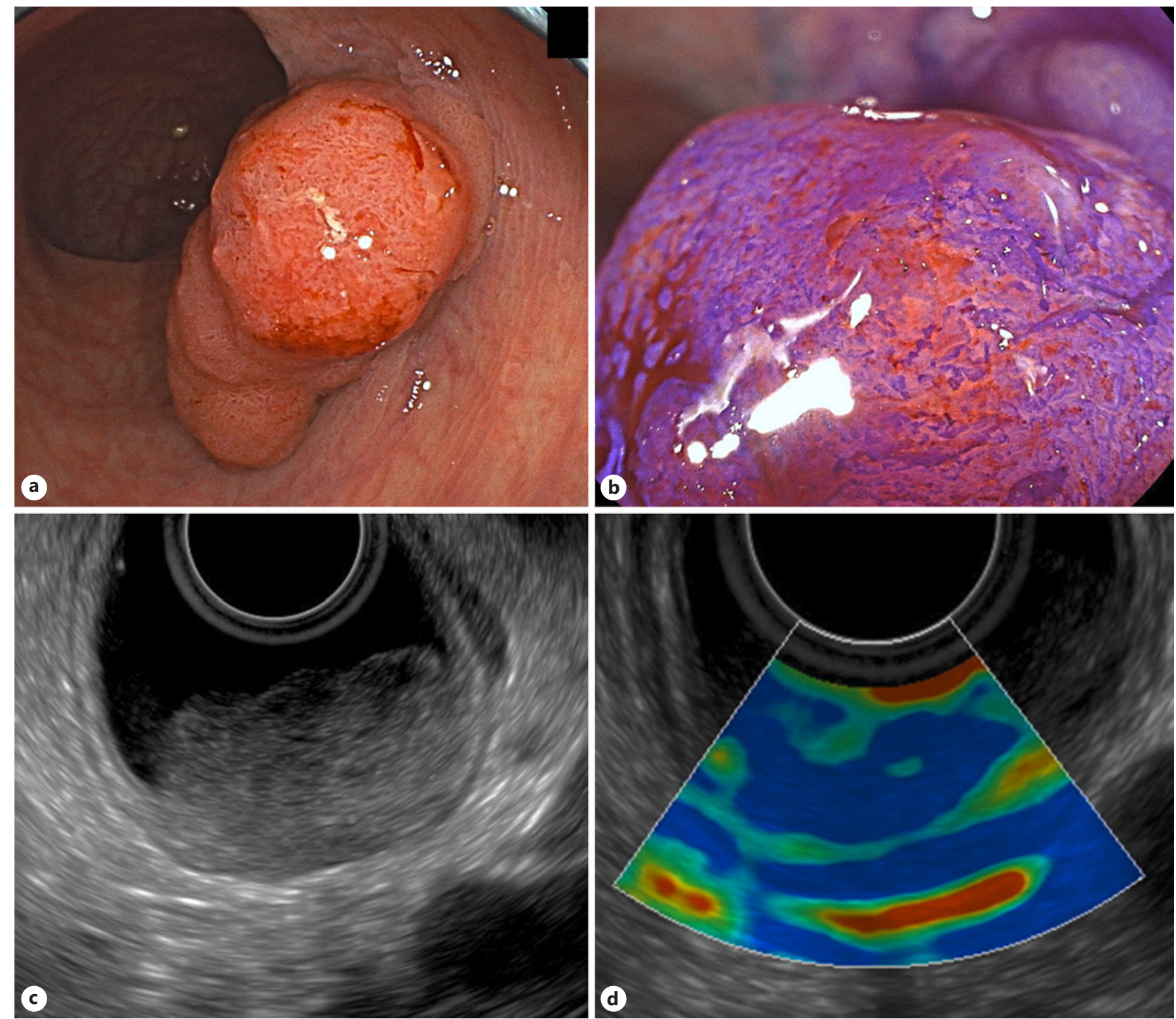

Fig. 4. A 30-mm, morphology type Is, lesion at the site of rectum with the final diagnosis of MP invasion confirmed by histopathological examination. a Findings on WLI showing a protruded lesion that is slightly reddish and bleeds easily. $\mathbf{b}$ Findings on MCE showing a VI high-grade pit pattern. c EUS findings showing invasion of the tumor into the third layer; of note, the demarcation with

the fourth layer is partially obscured. d EUS-EG findings showing that the tumor region is almost blue, indicative of an ES-CRN 2. $\mathrm{MP}$, muscularis propria; WLI, white light imaging; MCE, magnifying chromoendoscopy; EUS, endoscopic ultrasonography; EUSEG, endoscopic ultrasound elastography; ES-CRN, elastic score of colorectal neoplasm.

\section{Results}

\section{Derivation Study and Validation Study}

Thirty-one (33 lesions) and 50 (55 lesions) patients were enrolled in the derivation and validation studies, respectively (Fig. 3; Table 1). EUS-EG was performed on the

same day as MCE for 27 and 48 lesions for the derivation and the validation study, respectively, and within 2 weeks after MCE for the remaining 6 and 7, respectively. The echoendoscope was able to reach the target lesion in all cases, with no adverse events noted in both studies. Of the 33 and 55 lesions in the derivation and the validation 
Table 2. Diagnostic performance of each modality for dSM invasions or deeper lesions

\begin{tabular}{lcllll}
\hline Derivation study, $n=33$ & Sensitivity, \% (95\% CI) & Specificity, \% (95\% CI) & PPV, \% (95\% CI) & NPV, \% (95\% CI) & Accuracy, \% (95\% CI) \\
\hline EUS-EG* & $100(84.1-100)$ & $88.2(76.1-88.2)$ & $86.7(72.9-86.7)$ & $100(86.2-100)$ & $93.3(79.5-93.3)$ \\
MCE & $66.7(50.1-72.1)$ & $94.4(80.6-99.0)$ & $90.9(68.3-98.3)$ & $77.3(66.0-81.0)$ & $81.8(66.8-86.8)$ \\
EUS & $93.3(76.6-98.8)$ & $77.8(63.9-82.3)$ & $77.8(63.9-82.3)$ & $93.3(76.6-98.8)$ & $84.8(69.7-89.8)$ \\
\hline Validation study, $n=55$ & Sensitivity, \% (95\% CI) & Specificity, \% (95\% CI) & PPV, \% (95\% CI) & NPV, \% (95\% CI) & Accuracy, \% (95\% CI) \\
\hline EUS-EG** & $77.8(60.4-89.0)$ & $86.1(77.4-91.7)$ & $73.7(57.2-84.3)$ & $88.6(79.6-94.4)$ & $83.3(71.7-90.8)$ \\
MCE** & $84.2(68.8-92.6)$ & $91.4(83.1-96.0)$ & $84.2(68.8-92.6)$ & $91.4(83.1-96.0)$ & $88.9(78.0-94.8)$ \\
EUS** & $89.5(72.8-96.9)$ & $65.7(56.7-69.8)$ & $58.6(47.7-63.5)$ & $92.0(79.3-97.7)$ & $74.1(62.3-79.3)$ \\
\hline
\end{tabular}

$N$, number; EUS-EG, endoscopic ultrasound elastography; MCE, magnifying chromoendoscopy; EUS, endoscopic ultrasonography; $\mathrm{PPV}$, positive predictive value; NPV, negative predictive value; $\mathrm{CI}$, confidence interval. * Excluding 3 cases that are difficult to evaluate for EUS-EG. ${ }^{* *}$ Excluding 1 case that is difficult to evaluate for respective modality.

study, respectively, 13 and 18 lesions were surgically resected due to suspected dSM invasion based on WLI, MCE, and EUS findings. When the diagnosis for each modality was different, the MCE, which has the most evidence at present, was given priority. All lesions were treated by endoscopic or surgical resection within 1 month of the endoscopic procedures, with the final diagnosis confirmed by histopathological examination. Figure 4 shows a case presentation including MCE, EUS, and EUS-EG findings.

For each imaging modality, sensitivity, specificity, PPV, NPV, and accuracy of differentiating dSM or deeper lesions in the derivation, and validation studies were as follows: EUS-EG, 100/88.2/86.7/100/93.3\% and 77.8/ 86.1/73.7/88.6/83.3\%; MCE, 66.7/94.4/90.9/77.3/81.8\% and 84.2/91.4/84.2/91.4/88.9\%; and EUS, 93.3/77.8/ $77.8 / 93.3 / 84.8 \%$ and $89.5 / 65.7 / 58.6 / 92.0 / 74.1 \%$, respectively (Table 2). In the derivation study, EUS-EG had the highest accuracy, with MCE having the highest accuracy in the validation study. There was no statistical difference between the 3 modalities in either study. For EUS-EG diagnoses, a high confidence was obtained in 23/30 (76.7\%) and 51/54 (94.4\%) of cases in the derivation and the validation study, respectively. The distribution of the depth of invasion, confirmed by histopathological examination, for each ES-CRN classification (1 through 3), for MCE and EUS, is shown in Table 3.

Of the 33 and 55 lesions examined, 21 and 30 lesions included a type Is or Ip morphology, which are protruded lesions, in the derivation and the validation study, respectively. For these 21 and 30 protruded lesions, as well as for lesions with other types of morphology, diagnostic abili-
Table 3. Distribution of the depth of invasion, as confirmed by histopathological assessment, for each ES-CRN pattern (EUS-EG), MCE, and EUS

\begin{tabular}{|c|c|c|c|c|c|}
\hline \multirow{2}{*}{$\begin{array}{l}\text { Prediction of the } \\
\text { depth of lesions for } \\
\text { each modality }\end{array}$} & \multicolumn{5}{|c|}{$\begin{array}{l}\text { Derivation study/validation study } \\
\text { Histopathological assessment, } n\end{array}$} \\
\hline & $\mathrm{m}$ & sSM & $\mathrm{dSM}$ & MP & SS \\
\hline \multicolumn{6}{|l|}{ EUS-EG } \\
\hline \multicolumn{6}{|l|}{$\leq \mathrm{sSM}$} \\
\hline ES-CRN 1 & $14 / 28$ & $1 / 3$ & $0 / 2$ & $0 / 2$ & $0 / 0$ \\
\hline \multicolumn{6}{|l|}{$\geq \mathrm{dSM}$} \\
\hline ES-CRN 2 & $1 / 5$ & $1 / 0$ & $4 / 9$ & $4 / 3$ & $1 / 2$ \\
\hline ES-CRN 3 & $0 / 0$ & $0 / 0$ & $1 / 0$ & $3 / 0$ & $0 / 0$ \\
\hline \multicolumn{6}{|l|}{$M C E$} \\
\hline$\leq \mathrm{sSM}$ & $15 / 30$ & $2 / 2$ & $2 / 2$ & $2 / 1$ & $1 / 0$ \\
\hline$\geq \mathrm{dSM}$ & $1 / 3$ & $0 / 0$ & $5 / 10$ & $5 / 4$ & $0 / 2$ \\
\hline \multicolumn{6}{|l|}{ EUS } \\
\hline$\leq \mathrm{sSM}$ & $13 / 22$ & $1 / 2$ & $1 / 1$ & $0 / 1$ & $0 / 0$ \\
\hline$\geq \mathrm{dSM}$ & $3 / 10$ & $1 / 1$ & $6 / 11$ & $7 / 4$ & $1 / 2$ \\
\hline
\end{tabular}

$N$, number of cases; ES-CRN, elastic score of colorectal neoplasm; EUS-EG, endoscopic ultrasound elastography; MCE, magnifying chromoendoscopy; EUS, endoscopic ultrasonography; $\mathrm{m}$, intramucosal cancer; sSM, slight submucosal; dSM, deep submucosal; MP, muscularis propria; SS, subserosa.

ties for dSM or deeper lesions are reported for each imaging modality in the derivation and the validation study in Table 4. No significant difference in the diagnostic yield was observed between the 3 imaging modalities or morphology types. 
Table 4. Diagnostic performance of each diagnostic modality for dSM invasions or deeper lesions, including morphology type Is or Ip (I)/not including morphology type Is or Ip (II)

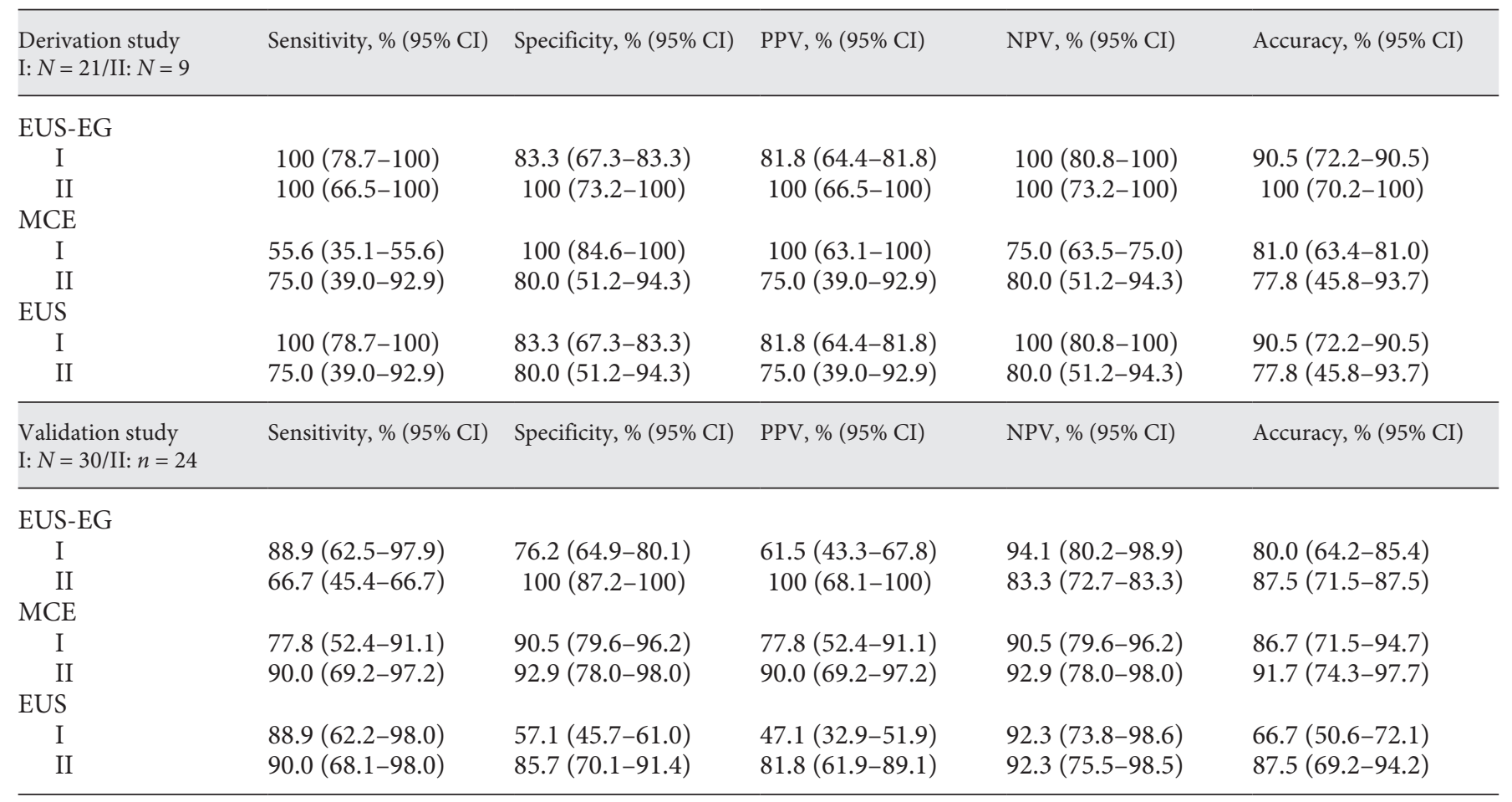

$N$, number; EUS-EG, endoscopic ultrasound elastography; MCE, magnifying chromoendoscopy; EUS, endoscopic ultrasonography; $\mathrm{PPV}$, positive predictive value; NPV, negative predictive value; CI, confidence interval.

In the derivation study, while MCE and EUS provided a useful diagnostic image for all lesions, the EUS-EG image was difficult to assess for 3 lesions as the ROI could not be adequately set, preventing the evaluation of the area immediately surrounding the lesion. Two of these lesions were located in the rectum and the other in the sigmoid colon, with a lesion size of 15,30 , and $36 \mathrm{~mm}$, respectively, and morphology type Is, Ip, and IIa + IIc, respectively. The depth of invasion on final histopathological diagnosis was SSM for 2 lesions and dSM in the third. In the validation study, each modality had one lesion that was difficult to assess: a lesion located on a fold for EUS and EUS-EG and a lesion with mucous adhesion for MCE.

\section{Reliability Examination in the Derivation Study}

In the reliability examination, inter-observer agreement was substantial to excellent between the 2 experts ( $\kappa$-coefficient: 0.84 and 0.78 for the first and second assessments, respectively) and moderate to substantial for the 2 non-experts ( $\kappa$-coefficient: 0.73 and 0.49 for the first and second assessments, respectively). Intra-observer agreement was substantial for the 2 experts ( $\kappa$-coefficients of 0.78 and 0.79 for the first and second assessments, respectively) and moderate to substantial for the 2 non-experts ( $\kappa$-coefficient of 0.68 and 0.52 for the first and second assessments, respectively). Sensitivity, specificity, PPV, NPV, and accuracy of diagnosis for dSM or deeper lesions and the number of EUS-EG diagnosis of high confidence for each observer (first assessment) are shown in Table 5.

\section{Discussion/Conclusion}

To our knowledge, this is the first study to evaluate the diagnostic ability and clinical efficacy of EUS-EG using a forward-viewing radial-array echoendoscope to determine the depth of invasion in colorectal neoplasms. Realtime tissue elastography is used to visualize tissue hardness $[34,35]$, producing a color overlay on the B-mode image with 256 levels of color gradation of tissue hard- 
Table 5. Reliability examination in the derivation study and the diagnostic performance of EUS-EG for dSM invasions or deeper lesions, as assessed by each observer

\begin{tabular}{llllll}
\hline & $\begin{array}{l}\text { Sensitivity, } \\
\%(95 \% \text { CI })\end{array}$ & $\begin{array}{l}\text { Specificity, } \\
\%(95 \% \text { CI })\end{array}$ & $\begin{array}{l}\text { PPV, } \\
\%(95 \% \text { CI })\end{array}$ & $\begin{array}{l}\text { NPV, } \\
\%(95 \% \text { CI })\end{array}$ & $\begin{array}{l}\text { Accuracy, } \\
\%(95 \% \text { CI })\end{array}$ \\
\hline $\begin{array}{l}\text { Expert A (T.Y.) } \\
\text { HC 19/30 }\end{array}$ & $92.3(73.9-98.5)$ & $82.4(68.3-87.1)$ & $80(64.0-85.4)$ & $93.3(77.4-98.7)$ & $86.7(70.7-92.1)$ \\
\hline $\begin{array}{l}\text { Expert B (H.S.) } \\
\text { HC 24/30 }\end{array}$ & $100(84.1-100)$ & $88.2(76.1-88.2)$ & $86.7(72.9-86.7)$ & $100(86.2-100)$ & $93.3(79.5-93.3)$ \\
\hline $\begin{array}{l}\text { Non-expert A (I.H.) } \\
\text { HC 16/30 }\end{array}$ & $100(82.9-100)$ & $58.8(45.8-58.8)$ & $65.0(53.9-65.0)$ & $100(77.8-100)$ & $76.7(61.9-76.7)$ \\
\hline $\begin{array}{l}\text { Non-expert B (K.Y.) } \\
\text { HC 18/30 }\end{array}$ & $92.3(73.3-98.6)$ & $58.8(44.3-63.6)$ & $63.2(50.2-67.5)$ & $90.9(68.5-98.3)$ & $73.3(56.9-78.8)$ \\
\hline
\end{tabular}

EUS-EG, endoscopic ultrasound elastography; PPV, positive predictive value; NPV, negative predictive value; CI, confidence interval; $\mathrm{HC}$, high confidence.

ness, from soft (red) to moderate (green) and hard (blue) [36]. Real-time tissue elastography was initially used to assess breast tissue, thyroid gland, prostate, and liver, using extracorporeal ultrasound. Similarly, EUS-based elastography is used to evaluate biliary and pancreatic diseases and digestive tract submucosal tumors [37]. For pancreatic lesions (classified into 5 elastic scores), EUSEG differentiates benign from malignant lesions, with a sensitivity and specificity ranging between 92.3-100\% and $67.0-80.0 \%$, respectively $[21,38]$. There have been reports of endorectal elastography providing valuable additional information for grayscale ultrasound imaging $[20,39]$. Although there are a few reports using elastography for colorectal tumors, there have been no reports of elastography using echoendoscopy for the tumors throughout the whole colorectum.

The diagnostic performance of MCE and EUS has previously been reported. For dSM or deeper lesions, pooled estimates from a meta-analysis of MCE, using the pit-pattern classification, reported a sensitivity and specificity of 81 and 95\%, respectively [33]. For EUS, when clear images were obtained, the accuracy for differentiating Tis and T1a cancers from T1b cancer was high, at approximately $90 \%[16,17,40]$. In our study, the diagnostic performance of EUS-EG was comparable to that of MCE and EUS. However, determining the depth of protruded-type colorectal neoplasms could be difficult using MCE and EUS, with these lesions often classified as "non-invasive" using MCE, even if they deeply invade the submucosal layer [18]. Protruded-type lesions are similarly difficult to image using EUS due to high rate of deep echo attenua- tion and more significant difficulty in vertically scanning semi-pedunculated and other types of protruded lesions [19]. Therefore, with MCE, there is a risk of considering the lesion to be shallower than the real depth, while with EUS, the depth may be estimated to be deeper than the real depth of the lesion. For these reasons, we included a range of lesion morphologies in our study, including Is or Ip which are protruded lesions. In our study, the decrease in diagnostic ability of each modality was not significant, even for the protruded lesions. For cases of misdiagnosis, compared to histopathological findings, the depth of invasion was lesser for 6 lesions using MCE and greater for 11 lesions using EUS and for 2 lesions using EUS-EG. Out of these, 5 lesions misdiagnosed by MCE were correctly diagnosed by EUS-EG. Six lesions misdiagnosed by EUS were correctly diagnosed by EUS-EG. This suggests that using EUS-EG may lead to a correct diagnosis of cases that have been misdiagnosed by conventional modalities. However, this study has small numbers, and our findings warrant confirmation by larger cohorts.

In the derivation study, 3 protruded lesions that were difficult to assess using EUS-EG were adequately visualized for diagnosis by MCE and EUS; however, in the validation study, all protruded lesions were adequately visualized by EUS-EG. It is possible that the reason for the noted difficulty in evaluation might reflect the learning curve rather than the morphology of the lesion. It has been shown that elastography results can vary widely depending on the skill level of the examiner in obtaining a stable image and in the selection and settings of the ROI for measurement [41]. In this study, some difficulties 
were experienced in appropriately setting the ROI for EUS-EG, indicating the continued need to advance technical aspects of EUS-EG. Similarly, EUS-EG can be cumbersome in other gastrointestinal regions due to effects of breathing and other movements. In our study, patient positioning and adjustments of the scope were used to control these influences during imaging in the colorectum.

Image-enhanced endoscopy of MCE is used with the Japan NBI Expert Team classification of the depth of invasion in colorectal neoplasms, with moderate inter-observer ( $\kappa$-coefficient, 0.52 ) and excellent intra-observer ( $\kappa$-coefficient, 0.88 ) agreements [42], based on the review of 199 lesions by 2 experienced colonoscopists. By comparison, a moderate to substantial intra- and inter-observer agreement was achieved by non-expert endoscopists using the ES-CRN classification. Based on these results, we consider that the ES-CRN classification was relatively simple to use and easy to understand, even for endoscopists unfamiliar with EUS-EG.

EUS is not usually performed for colorectal lesions due to difficulty with inserting a regular echoendoscope. However, the safety of advancing a forward-obliqueviewing upper echoendoscope to the sigmoid/left-sided colon for EUS imaging of benign and malignant colonic lesions has previously been reported [43]. Another report confirmed these results, with colorectal lesions being reached using a forward-viewing radial-array echoendoscope without adverse events, with the time to reach the lesion was similar to that of standard colonoscopy techniques [44]. Therefore, a forward-viewing radial-array echoendoscope can be safely used as the staging tool of choice for colon cancer. In our study, all lesions were similarly reached without adverse events.

The limitations of our study should be noted. First, this was a single-center case series; as such, selection bias cannot be denied. Second, all lesions were eligible for ESD or surgical resection, and there were no small lesions, which would have been better treated by EMR. Third, the lesions with scarring were excluded to reduce an impact of fibrosis. In fact, most intramucosal cancers were treated endoscopically in this study; there were few lesions with fibrosis in the result. Finally, the diagnostic ability of each modality was calculated excluding the cases that were difficult to assess. However, in the validation study, only one case was difficult to assess in each modality, respectively. Based on our findings, we propose that the ES-CRN classification (based on EUS-EG images) offer a potential adjunct for MCE and EUS to diagnose the depth of invasion in colorectal neoplasms. As well, with an accumulation of evidence, it might be possible to substitute EUS-EG, combined with the ES-CRN, for MCE or EUS in the future. We note cases which were difficult to assess or were misdiagnosed using MCE or EUS, with the diagnosis corrected using EUS-EG and EUS-EG and this with substantial interobserver agreement, even for non-experts. More accurate determination of submucosal invasion is valuable to inform appropriate treatment strategies. Further prospective studies should accumulate evidence of clinical usefulness of the ES-CRN classification for diagnosing the depth of colorectal neoplasms invasion.

\section{Acknowledgements}

We thank all members of the Department of Endoscopy, Nagoya University Hospital and Department of Gastroenterology and Hepatology, Nagoya University Graduate School of Medicine.

\section{Statement of Ethics}

This study was approved by the Research Ethics Board at Nagoya University Hospital (IRB No. 2015-0381) and registered in the University Hospital Medical Information Network Clinical Trials Registry (UMIN-CTR; study number UMIN000035902). Written informed consent was obtained from all patients. This study conforms to the provisions of the Declaration of Helsinki (as revised in Fortaleza, Brazil, October 2013).

\section{Conflict of Interest Statement}

The authors have no conflicts of interest to declare.

\section{Funding Sources}

The authors did not receive any funding.

\section{Author Contributions}

Study conception and design: Masaya Esaki and Takeshi Yamamura. Acquisition of data: Masaya Esaki, Yasuyuki Mizutani, Hiroto Suzuki, Takeshi Kuno, Kentaro Yamada, Issei Hasegawa, Takuya Ishikawa, Kazuhiro Furukawa, and Hiroki Kawashima. Analysis and interpretation of data: Masaya Esaki, Keiko Maeda, Tsunaki Sawada, Eri Ishikawa, Naomi Kakushima, Eizaburo Ohno, and Yoshiki Hirooka. Drafting of manuscript: Masaya Esaki. Critical revision: Masanao Nakamura. Final approval of the article: Mitsuhiro Fujishiro. 


\section{References}

1 Hurlstone DP, Sanders DS, Cross SS, Adam I, Shorthouse AJ, Brown S, et al. Colonoscopic resection of lateral spreading tumours: a prospective analysis of endoscopic mucosal resection. Gut. 2004 Sep;53(9):1334-9.

2 Luigiano C, Consolo P, Scaffidi MG, Strangio G, Giacobbe G, Alibrandi A, et al. Endoscopic mucosal resection for large and giant sessile and flat colorectal polyps: a single-center experience with long-term follow-up. Endoscopy. 2009 Aug;41(10):829-35.

3 Nakajima T, Saito Y, Tanaka S, Iishi H, Kudo $\mathrm{S}$, Ikematsu H, et al. Current status of endoscopic resection strategy for large, early colorectal neoplasia in Japan. Surg Endosc. 2013 March;27(9):3262-70.

4 Fujimori T, Kawamata H, Kashida H. Precancerous lesions of the colorectum. J Gastroenterol. 2001 Sep;36(9):587-94.

5 Kitajima K, Fujimori T, Fujii S, Takeda J, Ohkura Y, Kawamata H, et al. Correlation between lymph node metastasis and depth of submucosal invasion in submucosal invasive colorectal carcinoma: a Japanese collaborative study. J Gastroenterol. 2004 Jun;39(6): 534-43.

6 Tanaka S, Haruma K, Teixeira CR, Tatsuta S, Ohtsu N, Hiraga Y, et al. Endoscopic treatment of submucosal invasive colorectal carcinoma with special reference to risk factors for lymph node metastasis. J Gastroenterol. 1995 Dec;30(6):710-7.

7 Nusko G, Mansmann U, Partzsch U, Altendorf-Hofmann A, Groitl H, Wittekind C, et al. Invasive carcinoma in colorectal adenomas: multivariate analysis of patient and adenoma characteristics. Endoscopy. 1997;29(7):62631.

8 Sakuragi M, Togashi K, Konishi F, Koinuma K, Kawamura Y, Okada M, et al. Predictive factors for lymph node metastasis in T1 stage colorectal carcinomas. Dis Colon Rectum. 2003 Dec;46(12):1626-32.

9 Singh R, Nordeen N, Mei SL, Kaffes A, Tam W, Saito Y. West meets East: preliminary results of narrow band imaging with optical magnification in the diagnosis of colorectal lesions: a multicenter Australian study using the modified Sano's classification. Dig Endosc. 2011 May;23(1):126-30.

10 Yoshida N, Yagi N, Yanagisawa A, Naito Y. Image-enhanced endoscopy for diagnosis of colorectal tumors in view of endoscopic treatment. World J Gastrointest Endosc. 2012 Dec; 4(12):545-55

11 Sano Y, Hirata D, Saito Y. Japan NBI expert team classification: narrow-band imaging magnifying endoscopic classification of colorectal tumors. Dig Endosc. 2018 Apr; 30(4):543-5.

12 Kudo S, Rubio CA, Teixeira CR, Kashida H, Kogure E. Pit pattern in colorectal neoplasia: endoscopic magnifying view. Endoscopy. 2001 Apr;33(4):367-73.
13 Wada Y, Kashida H, Kudo SE, Misawa M, Ikehara N, Hamatani S. Diagnostic accuracy of pit pattern and vascular pattern analyses in colorectal lesions. Dig Endosc. 2010 June; 22(3):192-9.

14 Sakamoto T, Nakajima T, Matsuda T, Murakami $\mathrm{Y}$, Ishikawa $\mathrm{H}$, Yao $\mathrm{K}$, et al. Comparison of the diagnostic performance between magnifying chromoendoscopy and magnifying narrow-band imaging for superficial colorectal neoplasms: an online survey. Gastrointest Endosc. 2018 May;87(5):1318-23.

15 Saitoh Y, Obara T, Einami K, Nomura M, Taruishi $\mathrm{M}$, Ayabe T, et al. Efficacy of high-frequency ultrasound probes for the preoperative staging of invasion depth in flat and depressed colorectal tumors. Gastrointest Endosc. 1996 July;44(1):34-9.

16 Hurlstone DP, Brown S, Cross SS, Shorthouse AJ, Sanders DS. High magnification chromoscopic colonoscopy or high frequency 20 $\mathrm{MHz}$ mini probe endoscopic ultrasound staging for early colorectal neoplasia: a comparative prospective analysis. Gut. 2005 Nov; 54(11):1585-9.

17 Haruki S, Kobayashi K, Yokoyama K, Sada M, Koizumi W. Comparison of diagnostic accuracies of various endoscopic examination techniques for evaluating the invasion depth of colorectal tumors. Gastroenterol Res Pract. 2012 Oct;2012:621512.

18 Matsuda T, Fujii T, Saito Y, Nakajima T, Uraoka T, Kobayashi N, et al. Efficacy of the invasive/non-invasive pattern by magnifying chromoendoscopy to estimate the depth of invasion of early colorectal neoplasms. Am J Gastroenterol. 2008 Sept;103(11):2700-6.

19 Mukae M, Kobayashi K, Sada M, Yokoyama K, Koizumi W, Saegusa M. Diagnostic performance of EUS for evaluating the invasion depth of early colorectal cancers. Gastrointest Endosc. 2015 March;81(3):682-90.

20 Waage JE, Havre RF, Odegaard S, Leh S, Eide GE, Baatrup G. Endorectal elastography in the evaluation of rectal tumours. Colorectal Dis. 2011;13(10):1130-7.

21 Giovannini M, Hookey LC, Bories E, Pesenti C, Monges G, Delpero JR. Endoscopic ultrasound elastography: the first step towards virtual biopsy? Preliminary results in 49 patients. Endoscopy. 2006 Apr;38(4):344-8.

22 Zhu QL, Jiang YX, Liu JB, Liu H, Sun Q, Dai $\mathrm{Q}$, et al. Real-time ultrasound elastography: its potential role in assessment of breast lesions. Ultrasound Med Biol. 2008 Aug;34(8): 1232-8.

23 Alam F, Naito K, Horiguchi J, Fukuda H, Tachikake T, Ito K. Accuracy of sonographic elastography in the differential diagnosis of enlarged cervical lymph nodes: comparison with conventional B-mode sonography. Am J Roentgenol. 2008 Aug;191(2):604-10.
24 Dighe M, Bae U, Richardson ML, Dubinsky TJ, Minoshima S, Kim Y. Differential diagnosis of thyroid nodules with US elastography using carotid artery pulsation. Radiology. 2008;248(2):662-9.

25 Saftoiu A, Vilmann P, Gorunescu F, Ghonea DI, Gorunescu M, Ciurea T, et al. Neural network analysis of dynamic sequences of EUS elastography used for the differential diagnosis of chronic pancreatitis and pancreatic cancer. Gastrointest Endosc. 2008;68:1086-94.

26 Pallwein L, Mitterberger M, Struve P, Pinggera G, Horninger W, Bartsch G, et al. Realtime elastography for detecting prostate cancer: preliminary experience. BJU Int. $2007 \mathrm{Jul}$; 100(1):42-6.

27 Mezzi G, Arcidiacono PG, Carrara S, Boemo C, Testoni PA. Elastosonography in malignant rectal disease: preliminary data. Endoscopy. 2007 Apr;39(4):375.

28 Zhihui F, Yue C, Zhongyi Z, Rongjie L, Song $\mathrm{W}$, Kun Y. Shear wave elastography in rectal cancer staging, compared with endorectal ultrasonography and magnetic resonance imaging. Ultrasound Med Biol. 2019 Jul;45(7): 1586-93.

29 Gao L, Parker KJ, Lerner RM, Levinson SF. Imaging of the elastic properties of tissue: a review. Ultrasound Med Biol. 1996;22(8): 959-77.

30 Ophir J, Cespedes I, Garra B, Ponnekanti H, Huang Y, Maklad N. Elastography: ultrasonic imaging of tissue strain and elastic modulus in vivo. Eur J Ultrasound. 1996 Jan;3(1):4970.

31 Landis JR, Koch GG. The measurement of observer agreement for categorical data. Biometrics. 1997 Mar;33(1):159-74.

32 Hewett DG, Kaltenbach T, Sano Y, Tanaka S, Saunders BP, Ponchon T, et al. Validation of a simple classification system for endoscopic diagnosis of small colorectal polyps using narrow-band imaging. Gastroenterology. 2012 Sep;143(3):599-e1.

33 Backes Y, Moss A, Reitsma JB, Siersema PD Moons LM. Narrow band imaging, magnifying chromoendoscopy and gross morphological features for the optical diagnosis of $\mathrm{T} 1$ colorectal cancer and deep submucosal invasion: a systematic review and meta-analysis. Am J Gastroenterol. 2017 Jan;112(1):54-64.

34 Ueno E, Tohno E, Soeda S, Asaoka Y, Itoh K, Bamber JC, et al. Dynamic tests in real-time breast echography. Ultrasound Med Biol. 1988;14(Suppl 1):53-7.

35 Shiina T, Doyley MM, Bamber JC. Strain imaging using combined RF and envelope autocorrelation processing. Proc IEEE Ultrason Symp. 1996;2:1331-4.

36 Săftoiu A, Vilman P. Endoscopic ultrasound elastography: a new imaging technique for the visualization of tissue elasticity distribution. $J$ Gastrointestin Liver Dis. 2006 June; 15(2): 161-5. 
37 Dietrich CF, Săftoiu A, Jenssen C. Real time elastography endoscopic ultrasound (RTEEUS), a comprehensive review. Eur J Radiol. 2014 March;83(3):405-14.

38 Giovannini M, Thomas B, Erwan B, Christian P, Fabrice C, Benjamin E, et al. Endoscopic ultrasound elastography for evaluation of lymph nodes and pancreatic masses: a multicenter study. World J Gastroenterol. 2009 Apr;15(13):1587-93.

39 Andrea G, Marco B, Marco M. Feasibility of real-time strain elastography in colonic diseases. J Ultrasound. 2014 Dec;17(4):321-30.
40 Saitoh Y, Watari J, Fujiya M, Kohgo Y. Highfrequency ultrasound probes in the evaluation of colorectal neoplasia. Digestive Endoscopy. 2001 July;13(s1):S14-8.

41 Nakashima K, Mizutou A, Sakurai S. Auto strain ratio system for the quality control of breast strain elastography. J Med Ultrasonics. 2018 Apr;45(2):261-8.

42 Komeda Y, Kashida H, Sakurai T, Asakuma Y, Tribonias G, Nagai T, et al. Magnifying narrow band imaging (NBI) for the diagnosis of localized colorectal lesions using the Japan NBI Expert Team (JNET) classification. Oncology. 2017 Dec;93(Suppl 1):49-54.
43 Bhutani MS, Nadella P. Utility of an upper echoendoscope for endoscopic ultrasonography of malignant and benign conditions of the sigmoid/left colon and the rectum. Am J Gastroenterol. 2001 Dec;96(12):3318-22.

44 Kongkam P, Linlawan S, Aniwan S, Lakananurak N, Khemnark S, Sahakitrungruang C, et al. Forward-viewing radial-array echoendoscope for staging of colon cancer beyond the rectum. World J Gastroenterol. 2014 Mar; 20(10):2681-7. 